\section{GRP-138 PHARMACEUTICAL INTERVENTIONS IN THE SETTING OF LIPIDS IN PAEDIATRIC PARENTERAL NUTRITION}

doi:10.1136/ejhpharm-2013-000276.138

C Alarcon-Payer, J Pérez Morales, C Valencia Soto, P Vallecillo Capilla, E Puerta Garcia, MA Calleja Hernández. Hospital Universitario Virgen de las Nieves, Servicio de Farmacia, Granada, Spain

Background The preparation of paediatric parenteral nutrition (PPN) is subject to a great deal of variability in clinical practise. Standardization in the process is indispensable to ensure stability and improve patient safety. The pharmacist plays an essential role in the proper preparation of all-in-one PPN, and in interventions to avoid problems associated with instability.

Purpose The 2008 Spanish consensus on the preparation of parenteral nutrient mixtures established a minimum lipid percentage of $1.5 \%$. The aim of this study was to detect PPN prescriptions with a lipid percentage below $1.5 \%$, considered the safe limit for lipid emulsion stability in ternary mixtures.

Materials and Methods Observational retrospective descriptive study of PPN requirements in a tertiary level hospital. It was conducted between September 2011 and June 2012. Prescriptions in which the lipid composition of the PPN was less than $1.5 \%$ of the mixture were reviewed. In all cases, the intervention involved having the pharmacist contact the prescribing physician. Proposed alternatives to preserve the stability were: a) increase the proportion of lipid; b) exclude lipids from the mixture; or c) decrease the mixture volume.

Results A total of 107 interventions were made during this period. $100 \%$ of the physicians contacted accepted the intervention. In $81.3 \%$ of cases they agreed to increase the weight of lipids by an average of $1 \mathrm{~g}$; the median was $0.8 \mathrm{~g}$. The $18.7 \%$ remaining cases chose to exclude lipids from the mixture during the first few days, and add lipids gradually thereafter. In these cases the initial average of lipids was $1.1 \mathrm{~g}$, and the median $0.8 \mathrm{~g}$. In no case was the total volume changed.

Conclusions The results support the role of the pharmacist in the proper management of paediatric PNN, and in ensuring the quality and safety of the mixture. The results also support the importance of pharmacist-physician collaboration.

No conflict of interest.

\section{GRP-139 PHARMACIST INTERVENTIONS IN THE EMERGENCY ROOM OF A TERTIARY-LEVEL HOSPITAL}

doi:10.1136/ejhpharm-2013-000276.139

H Mateo, M Giménez Ramos, P Nieto, FD Fernández Ginés, JJ Fernández Ávila. Torrecárdenas Hospital, Pharmacy, Almería, Spain

Background The presence of a full-time pharmacist in the ER is well established and acknowledged in many institutions to be of great value.

Purpose To analyse and assess the clinical impact of the pharmacist's interventions in the ER of a tertiary-level teaching institution

Materials and Methods Patients $>65$ years-old on $>4$ medications were included. Drug-related problems (DRP) were classified according to: (a) therapeutic group; (b) intensity: mild, moderate, severe, and very severe; and (c) type (from Martí et al, 2005): indication, safety, adherence, and effect. Patients were monitored for up to $72 \mathrm{~h}$ upon subsequent admission to a ward.

Results 111 patients were included, $70.2 \%$ male, median age 78.9 years [65-94]. 34 pharmaceutical recommendations were made (one for every 3.2 patients included), of which $85.2 \%$ were subsequently accepted. The largest number of interventions concerned antithrombotics, followed by antihypertensives $(29.4$ and $5.4 \%$ of the interventions, respectively). $18.01 \%$ met an indication $(81.1 \%$ of them were off-label conditions), $5.4 \%$ were to do with safety (mostly overdose), 1.8\% concerned compliance and $0.9 \%$ involved under-dosing. Overall, $75.86 \%$ of the interventions had a mild-tomoderate impact, whilst $17.25 \%$ were moderate-to-severe (involving mainly anticoagulants), and $6.89 \%$ (immunosuppressants) were serious or very serious.

Conclusions Real time support to physicians and nurses in the ER allowed the early detection of potential DRPs in one third of the patients. Cardiovascular disease required almost two thirds of interventions, with antithrombotic drugs as the drugs mainly involved (1/10 patients in need of thromboprophylaxis lacked it). However, the clinical impact was minimised by the short time spent in the unit (slightly under one day), and by the further revision of their medicines upon admission to a ward. In addition, the narrower the therapeutic range of the drugs involved (such as immunosuppressants), the more important is the timely contribution of the pharmacist. In conclusion, the presence of a full-time pharmacist in the ER would reduce DRPs by an exhaustive assessment of pharmacotherapeutic needs, which is particularly important for older or polymedicated patients.

No conflict of interest.

\section{GRP-140 PHARMACIST INVOLVEMENT IN CYTOSTATIC DOSES: IN AN OBESE POPULATION}

doi:10.1136/ejhpharm-2013-000276.140

A Izquierdo, S Martínez, A Alfaro, M Merchante, L Sanchez-Rubio, A Zorzano, MP Aibar, A Serrano, L Carrillo, MF Hurtado. San Pedro Hospital, Hospital Pharmacy, Logroño, Spain

Background The dosing of chemotherapy according to the body surface area (BSA) in obese adult patients, who present a BSA above $2 \mathrm{~m}^{2}$, is usually set to an established BSA maximum limit of $2 \mathrm{~m}^{2}$. The recent publication (April 2012): 'Appropriate chemotherapy dosing for obese adult patients with cancer' in the American Society of Clinical Oncology Clinical Practice Guidelines (ASCO), considers the benefit of full dosing, without adjusting to a maximum BSA, especially when the goal of treatment is curative.

Purpose To suggest recommendations for adult obese people according to the current ASCO guidelines and evaluate the medical prescribers' level of acceptance.

Materials and Methods Prospective observational study of all patients from the oncology, haematology and palliative care services receiving chemotherapy from April to June 2012. In those obese patients where the cytostatic dose was adjusted to BSA $2 \mathrm{~m}^{2}$, it was recommended to dose according to their actual BSA. The article was disseminated in these services and a spreadsheet was created to record the level of acceptance from the medical prescriber in each of the clinic units.

Results 368 patients (56\% female) were included: $82.3 \%$ from the oncology service, $16.6 \%$ from haematology and $1 \%$ from the palliative care service. The average \pm standard deviation age was $61.2 \pm 1.6$ years, $69.3 \pm 14.1 \mathrm{Kg}$ and $1.7 \pm 0.2 \mathrm{~m}^{2}$. The number of patients with a BSA above $2 \mathrm{~m}^{2}$ was 26 (7\%): $50 \%$ were from the oncology service and none from the palliative service.

Recommendations were made in 17 (65\%) of the patients with a BSA $>2 \mathrm{~m}^{2}$, of which the haematology service was the largest cohort (58\%). The acceptance level was 53\% (66.6\% haematology service). Recommendations were not made to $35 \%$ ( $66.6 \%$ oncology service) because the treatments were started after the article had been disseminated and full doses were prescribed.

The use of full doses was well tolerated by all patients, no adverse outcomes were observed of the use of greater doses of chemotherapy. 